\title{
Estados inmaduros de Ancognatha ustulata (Coleoptera: Melolonthidae: Dynastinae: Cyclocephalini)
}

\author{
Immature stages of Ancognatha ustulata (Coleoptera: Melolonthidae: Dynastinae: \\ Cyclocephalini)
}

\author{
Jhon César Neita-Moreno ${ }^{1}$ y Miguel Ángel Morón ${ }^{2 *}$ \\ ${ }^{1}$ Grupo de Sistemática de Insectos-Agronomía. Museo Entomología UNAB. Universidad Nacional de Colombia. Ciudadela Universitaria Bloque 500, \\ Museo de Entomología 220, Bogotá, Colombia. \\ ${ }^{2}$ Departamento de Biología de Suelos, Instituto de Ecología, A. C. Apartado postal 63, 91000, Xalapa, Veracruz, México. \\ "Correspondencia: miguel.moron@inecol.edu.mx
}

\begin{abstract}
Resumen. Se describen y se ilustran por primera vez la larva de tercer estadio y la pupa de Ancognatha ustulata Burmeister, 1847 (Coleoptera: Melolonthidae: Dynastinae: Cyclocephalini). Se aportan datos sobre la biología de la especie y su distribución en Colombia. Se proporciona una clave para la identificación de las larvas de tercer estadio conocidas de las especies del género Ancognatha Erichson.
\end{abstract}

Palabras clave: Scarabaeoidea, larva, pupa, taxonomía, Colombia.

\begin{abstract}
The third instar larva and pupa of Ancognatha ustulata Burmeister, 1847 (Coleoptera: Melolonthidae: Dynastinae: Cyclocephalini) are described and illustrated for the first time. New data on larval biology and distribution of the species in Colombia are included. A key to the known third stage larvae of Ancognatha Erichson is provided.
\end{abstract}

Key words: Scarabaeoidea, larva, pupa, taxonomy, Colombia.

\section{Introducción}

El género Ancognatha Erichson, 1847 reúne 22 especies que se distribuyen desde Arizona y Nuevo México, en el sur de los Estados Unidos, a Ecuador, Perú y Bolivia (Endrödi, 1966; 1985; Ratcliffe, 1992; 2003; Krajcik, 2005; Pardo-Locarno et al., 2006). En Colombia se han registrado 10 de estas especies (Restrepo-Giraldo et al., 2003; Pardo-Locarno et al., 2006): A. atacazo Kirsch, 1885, A. castanea Erichson, 1847, A. horrida Endrödi, 1967, A. humeralis Burmeister, 1847, A. lutea Erichson, 1847, A. matilei Dechambre, 2000, A. scarabaeoides Erichson, 1847, A. ustulata Burmeister, 1847, A. veliae Pardo-Locarno et al., 2006, y A. vulgaris Arrow, 1911. Con mucha frecuencia las larvas de $A$. scarabaeoides han sido consideradas como plaga rizófaga que causa gran perjuicio económico en varios cultivos, especialmente en papa (Solanum tuberosum L., Solanaceae) (Otoya, 1945; Ruiz y Posada, 1985; Pardo-Locarno et al., 1993; 2003; Londoño y Pérez, 1994; Montoya y Madrigal, 1994; Sánchez-Gutiérrez y Vázquez-Acosta, 1996; Vallejo, 1997; Restrepo-Giraldo y López-Ávila, 2000). Debido

Recibido: 15 octubre 2007; aceptado: 12 febrero 2008 a que no se han publicado trabajos taxonómicos o de morfología comparada con las larvas de Ancognatha y otros géneros afines en Colombia, ni se conoce con precisión la distribución geográfica de las 10 especies, y no se cuenta con suficiente información sobre sus hábitos, es muy posible que en ocasiones se confundan las larvas y, erróneamente se atribuyan los daños agrícolas a una sola especie o, en otro caso, la plaga verdadera pudiera corresponder a otro género de Melolonthidae. Considerando lo anterior, el presente trabajo tiene como objetivos: describir los estados inmaduros de Ancognatha ustulata, compararlos con los inmaduros de otras 3 especies del género y elaborar una clave para la identificación de las larvas de tercer estadio del género Ancognatha hasta el momento conocidas.

\section{Materiales y métodos}

Las larvas descritas en el presente artículo fueron capturadas durante el desarrollo del proyecto "Taxonomía y distribución de chisas (Coleoptera: Melolonthidae) asociadas a 5 cultivos de importancia agrícola en Cundinamarca, Colombia" entre los años 2003-2006. El $50 \%$ de los ejemplares inmaduros obtenidos en el campo 
se fijaron en solución de Pampel (Stehr, 1987), y el otro $50 \%$ se utilizó para obtener exuvias, pupas y adultos en cautiverio y verificar su identidad. Cada larva se colocó en un recipiente de $500 \mathrm{ml}$ de capacidad con tierra y trigo germinado para que culminase su ciclo de desarrollo. Los recipientes se situaron en un cuarto con temperatura y humedad regulada durante 12 meses. De las muestras se tomaron 6 larvas de tercer estadio para realizar las descripciones e ilustraciones, y entre 10 y 14 ejemplares para obtener las dimensiones de la cápsula cefálica y la longitud del cuerpo, con ayuda de un ocular micrométrico o un calibrador milimétrico. Las observaciones, descripciones e ilustraciones se realizaron con la ayuda de un estereomicroscopio.

Los caracteres y la terminología utilizada en las descripciones está basada en las propuestas de Böving (1942) Ritcher (1966) y Morón (1986, 1987). Los ejemplares de adultos y estados inmaduros se depositaron en la colección del Museo de Entomología, Universidad Nacional de Colombia, Bogotá (UNAB). Para precisar la distribución de A. ustulata en Colombia se estudiaron las siguientes colecciones: Museo Entomológico Francisco Luis Gallego, Universidad Nacional de Colombia, sede Medellín (MEFLG); Instituto de Ciencias Naturales - Universidad Nacional de Colombia, sede Bogotá (ICNMHN-CO); Centro Internacional de Agricultura Tropical, Palmira-Valle del Cauca (CE-CIAT); Universidad del Quindío, Armenia-Quindío (CE-UQ); Universidad del Valle, Calí-Valle del Cauca (MEUV); Museo de Ciencias Naturales "Gonzalo Andrades", Universidad Pedagógica y Tecnológica de Tunja, Boyacá (MHN-GA); Facultad de Agronomía, Universidad Pedagógica y Tecnológica de Tunja, Boyacá (CE-FA); Universidad de Cundinamarca, Fusagasuga-Cundinamarca (CE-UC); Universidad de Antioquia, Medellín-Antioquia (CE-UDA); Universidad Pedagógica Nacional, Bogotá-Cundinamarca (CEUPN); Facultad de Agronomía, Universidad Nacional de Colombia, Bogotá-Cundinamarca (UNAB); Universidad Tecnológica del Chocó "Diego Luis Córdoba", QuibdóChocó (MHN-CE); Instituto Alexander Von Humboldt, Villa de Leyva-Boyacá (IAVH); y Colección Particular Jhon César Neita M. Bogotá-Cundinamarca (CPJCN).

\section{Los estados inmaduros de Ancognatha Erichson}

Ritcher (1966) describió la larva de tercer estadio de Ancognatha manca con ejemplares de Arizona, EUA y Ramírez-Salinasycolaboradores(2004)describieronlalarva y la pupa de $A$. sellata con una muestra de Chiapas, México. Con base en tales estudios y en nuestras observaciones, es posible indicar que las larvas de Ancognatha se diferencian de los otros géneros de Cyclocephalini y otros Dynastinae por la siguiente combinación de caracteres: sedas frontales anterior, posteriores y externas ausentes. Ocelos presentes, no pigmentados (excepto en $A$. manca, que los presenta pigmentados). Haptomerum de la epifaringe bidentado (excepto en $A$. manca que lo tiene entero). Lacinia con 3 unci separados en la base; área estriduladora maxilar con proceso ventral anterior y 7-12 dientes truncados. Último artejo antenal con 2 áreas sensoriales dorsales y 2 ventrales. Raster sin palidia. Abertura anal ligeramente curvada.

\section{Descripciones}

Ancognatha ustulata Burmeister, 1847 (Figs. 1-16)

Larva de tercer estadio. Aspecto general: Longitud dorsal total aproximada: $58-60 \mathrm{~mm}$; cabeza pardo obscuro a pardo rojizo. Cuerpo blanco cremoso, casi cilíndrico, regiones dorsales con numerosas sedas de diferentes longitudes, regiones laterales y ventrales con sedas escasas. Escleromas protorácicos amarillo rojizo; placas respiratorias pardo
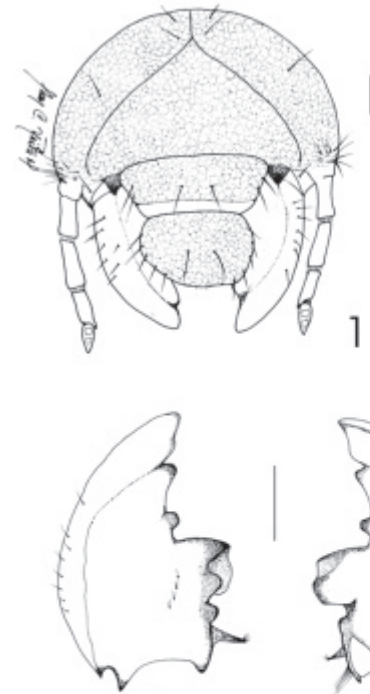

3

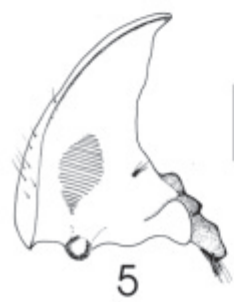

5
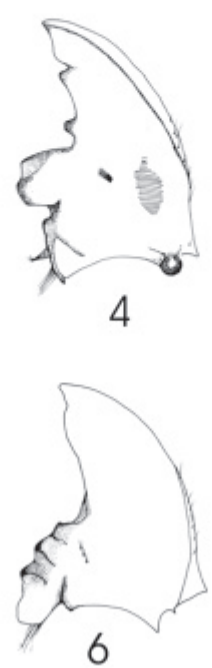

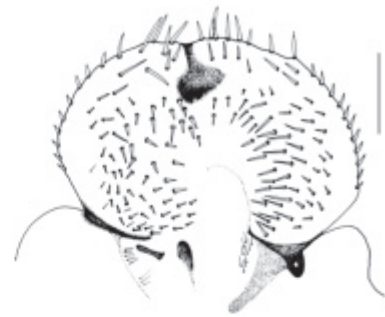

2
Figuras 1-8. Ancognatha ustulata, larva de tercer estadio. 1, aspecto frontal de la cápsula cefálica; 2, epifaringe; 3, vista dorsal de la mandíbula izquierda; 4 , vista ventral de la mandíbula izquierda; 5 , vista ventral de la mandíbula derecha; 6 , vista ventral de la mandíbula derecha; 7, vista dorsal de la maxila izquierda; 8, vista mesial del ápice de la maxila derecha. Escala: $1 \mathrm{~mm}$. 
Clave para la identificación de las larvas de tercer estadio del género Ancognatha Erichson:

1. Haptomerum de la epifaringe entero. Ocelos pigmentados. Suroeste de EUA y nor-occidente de México ............ A. manca LeConte

1' Haptomerum de la epifaringe bidentado. Ocelos no pigmentados....

2. Placas respiratorias de los 8 segmentos abdominales de igual tamaño. Acantoparia con 6-10 sedas cortas espiniformes. Ángulos frontales anteriores con 2 sedas. Sureste de México a norte de Nicaragua................................................................... A. sellata Arrow

2’ Placas respiratorias del primer segmento abdominal más pequeñas que las de los segmentos II-VIII...... .3

3. Borde lateral posterior izquierdo de la epifaringe redondeado. Laeophoba con 7 sedas delgadas y cortas. Unci 2 y 3 separados por la misma distancia entre el 1 y 2; unci dispuestos en el mismo plano. Panamá a Ecuador. ........................A. scarabaeoides Erich

3' Borde lateral posterior izqyuierdo de la epifaringe angulado. Laeophoba con 18 sedas delgadas y cortas. Unci 2 y 3 separados 2 veces la distancia entre el 1 y 2 ; unci no dispuestos en el mismo plano. Colombia, Ecuador y Venezuela....... A. ustulata Burmeister

obscuro; patas pardo-amarillas. Cabeza (Fig. 1). Anchura máxima de la cápsula cefálica: 6.5-7.0 mm. Superficie del cráneo finamente punteada y reticulada. Frente con 1 seda en cada uno de los ángulos frontales anteriores; sedas anteriores, posteriores y externas ausentes. El resto de la superficie craneal con 2 sedas dorsoepicraneales, 2 sedas epicraneales, y 8-9 sedas paraocelares a cada lado. Ocelli pequeños, bien definidos. Clípeo con 2 sedas centrales y 2 sedas laterales a cada lado. Labro ligeramente asimétrico, con 5 sedas laterales y 2 sedas centrales, sin sedas posteriores. Epifaringe (Fig. 2) con $0.49 \mathrm{~mm}$ de anchura y $0.35 \mathrm{~mm}$ de longitud. Borde lateral posterior izquierdo angulado. Proceso haptomeral prominente y bidentado. Acanthoparia formada por 9-12 sedas espiniformes cortas recurvadas. Corypha con 6 sedas delgadas y cortas. Acroparia formada por 17-18 sedas delgadas. Chaetoparia izquierda con 54-56 sedas. Chaetoparia derecha con 74-76 sedas. Dexiotorma delgada, con el extremo mesial aguzado. Laeotorma más corta que la dexiotorma; extremo mesial no aguzado, con pterotorma redondeada y pronunciada. Placa esclerosada grande y angulosa; cono sensorial ancho y prominente con 4 sensilias. Laeophoba consistente de 18 filamentos delgados y cortos ligeramente esparcidos entre el haptolechus y la laeotorma. Crepis fino, ligeramente esclerosado y bien definido. Pedium amplio. Mandíbula izquierda (Figs. 3-4). Área incisiva con 3 dientes $S_{1}+S_{2}$ fusionados, $\mathrm{S}_{3}$ separado por la escotadura postincisiva y $\mathrm{S}_{4}$ truncado, scrobis con 8 sedas largas y delgadas. Superficie dorsal con una línea de 7 sedas; acia bien desarrollada, alargada y triangular, con 3 sedas apicales cortas y delgadas. Superficie ventral con una área estriduladora ovalada formada por 24 surcos transversales; proceso ventral desarrollado, brustia con 16 sedas moderadamente largas y gruesas dispuestas en forma de "U"; 2 sedas basolatelares delgadas y moderadamente cortas. Área molar con 3 lóbulos, el primero más desarrollado que el segundo y el tercero. Mandíbula derecha (Figs. 5-6). Área incisiva con 3 dientes, $\mathrm{S}_{1}$ y $\mathrm{S}_{2}$ fusionados, y $\mathrm{S}_{3}$ situado después de la escotadura incisiva. Scrobis con 7-8 sedas delgadas y largas. Superficie dorsal con una línea de 7 sedas. Superficie ventral con área estriduladora ovalada formada por 28 surcos transversales; proceso ventral desarrollado; brustia con 8 sedas moderadamente largas y gruesas. Calx prominente, con 1 seda basolateral delgada y moderadamente corta. Área molar con 3 lóbulos, el primero más desarrollado. Maxila (Figs. 7-8). Cardo subtriangular. Estipe más largo que ancho. Galea y lacinia fusionadas en un mala. Galea con muchas sedas gruesas y 1 uncus bien desarrollado, rodeado por 7 heli gruesos. Lacinia con muchas sedas gruesas y delgadas mezcladas y 3 unci; el tercero de ellos separado de los otros unci por el doble de la distancia entre los 2 primeros. El primero y el tercer unci dispuestos en el mismo plano, y el segundo unci ligeramente divergente respecto a los otros. Palpos formados por 4 artejos. Área estriduladora maxilar (Fig. 9) con 9 dientes truncados y un proceso anterior. Labio con superficie anterolateral de la glosa con 28 sedas delgadas y largas; superficie central de la glosa con 25 sedas gruesas y cortas. Escleroma hipofaríngeo (Fig. 10) asimétrico, cóncavo mesialmente, con un proceso dorsal en el lado derecho. Lóbulo lateral derecho con 24 sedas cortas y delgadas. Lóbulo lateral izquierdo con 18 sedas delgadas y moderadamente largas, y una línea de 18 sedas moderadamente largas y gruesas rodean el escleroma con dirección hacía el fondo faríngeo; 2 sedas gruesas en la base del escleroma. Palpos labiales formados por 2 artejos, el segundo aproximadamente 2 veces más largo que el primero. Antenas largas, compuestas por 4 artejos, el artejo apical con 2 áreas sensoriales dorsales (Fig. 1) y 2 áreas sensoriales ventrales.

Tórax. Pronoto con 1 escleroma lateral de bordes obtusos, con 2 sedas delgadas y largas. Las placas respiratorias en forma de "C", largo $0.35 \mathrm{~mm}$, ancho $0.23 \mathrm{~mm}$ (Fig. 11), la distancia entre los lóbulos de la placa respiratoria 

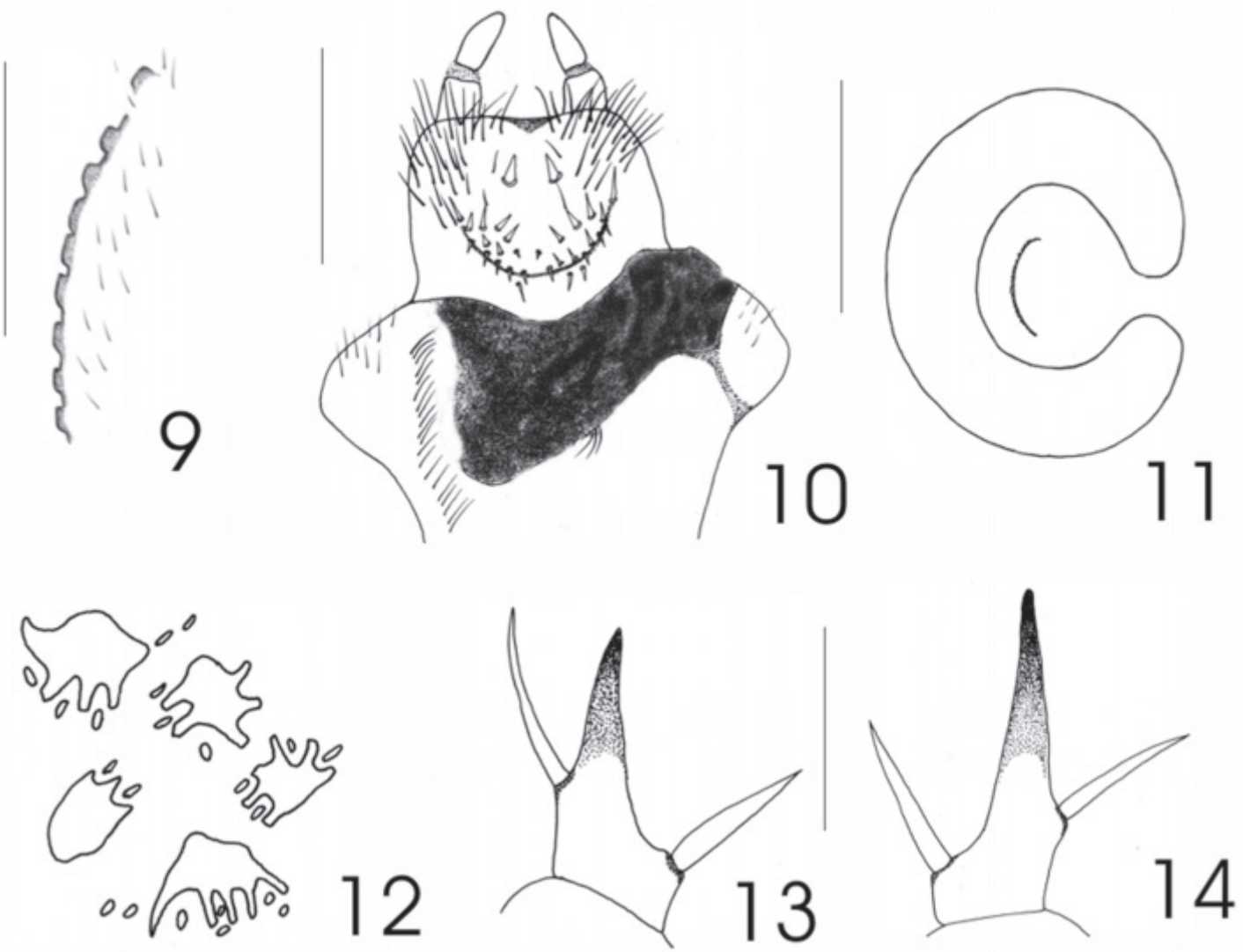

Figuras 9-14. Ancognatha ustulata, larva de tercer estadio. 9, detalle del órgano estridulador maxilar; 10, labio hipofaringe; 11, aspecto lateral de la placa respiratoria protorácica izquierda; 12 , detalle de las perforaciones de la placa respiratoria; 13, vista posterior del meso-tarsúngulo derecho; 14, vista posterior del meta-tarsúngulo izquierdo; Escala: $0.5 \mathrm{~mm}$, excepto en la fig. $10=1 \mathrm{~mm}$.

menor que el diámetro dorso-ventral de una bulla poco prominente; en un sector transversal la placa respiratoria tiene 27 perforaciones (promedio) de forma muy irregular, rodeadas de diminutos orificios (Fig. 12). Dorso del protórax con una hilera transversal de 8 sedas delgadas y largas; mesopreescudo con una hilera irregular de 6 sedas delgadas y largas; mesoescutelo con 4 sedas delgadas y largas; metapreescudo con 6 sedas largas y delgadas, y sin sedas cortas espiniformes; metaescudo con 4 sedas delgadas y largas, y 8 sedas cortas espiniformes; metaescutelo con 4 sedas delgadas y largas, y sin sedas cortas espiniformes. Patas. Pro- y metatarsúngulos más pequeños que los mesotarsúngulos, alargados con el ápice agudo, con 1 seda basal interna y 1 seda prebasal externa (Figs. 13-14).

Abdomen. Placa respiratoria del primer segmento abdominal más pequeña (largo $0.27 \mathrm{~mm}$, ancho $0.19 \mathrm{~mm}$ ) que las placas respiratorias de los segmentos II-VIII; las placas respiratorias de los segmentos II-VI son ligeramente más largas (largo $0.33 \mathrm{~mm}$, ancho $0.2 \mathrm{~mm}$ ) que las placas de los segmentos VII y VIII (largo $0.33 \mathrm{~mm}$, ancho 0.25 $\mathrm{mm})$; placas respiratorias regularmente recurvadas en forma de "C", la distancia entre los lóbulos de las placas respiratorias menor que el diámetro dorso-ventral de una bulla no prominente. Primer segmento abdominal: preescudo con 2 sedas delgadas y largas, y 4 sedas cortas, espiniformes; subescudo sin sedas; escudo con 12 sedas delgadas y largas, y 6 sedas cortas, espiniformes; escutelo con 12 sedas delgadas y largas, y 6 sedas cortas espiniformes; área respiratoria con 7 sedas delgadas y largas (Fig. 15). Segmento abdominal II: preescudo con 2 sedas delgadas y largas, y 14 sedas cortas, espiniformes; subescudo con 3 sedas delgadas y largas; escudo con 14 sedas delgadas y largas, y 23 sedas cortas, espiniformes; escutelo con 6 sedas delgadas y largas, y 28 sedas cortas espiniformes; área respiratoria con 7 sedas delgadas y largas. Segmento abdominal III: preescudo con 2 sedas delgadas y largas, y 29 sedas cortas, espiniformes; subescudo con 3 sedas largas y delgadas; escudo con 14 sedas delgadas y largas, y 35 sedas cortas, espiniformes; escutelo con 8 sedas delgadas y largas, y 46 sedas cortas espiniformes. Segmento abdominal IV: preescudo con 2 sedas delgadas y largas, y 36 sedas cortas, espiniformes; subescudo con 


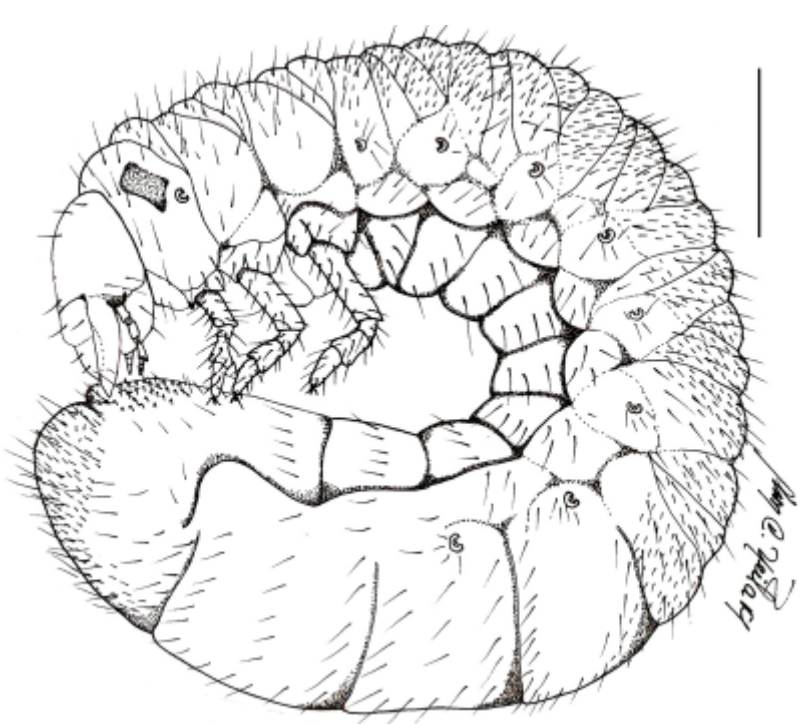

Figura 15. Ancognatha ustulata, larva de tercer estadio. Escala: $1 \mathrm{~cm}$.

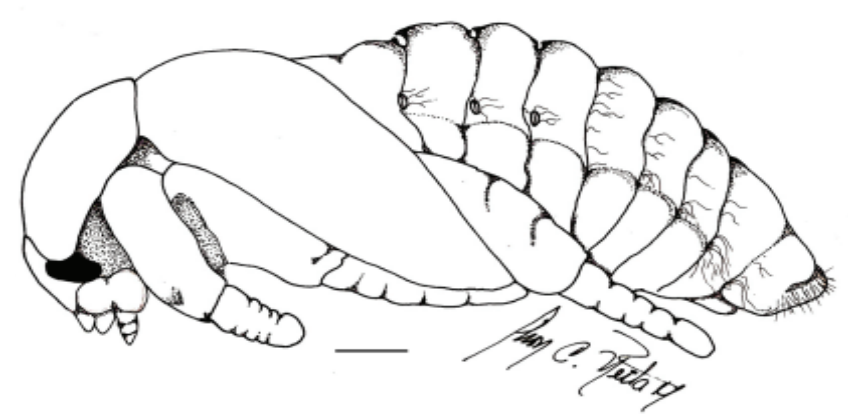

Figura 16. Ancognatha ustulata, pupa macho. Escala: $1 \mathrm{~cm}$.

3 sedas largas y delgadas; escudo con 12 sedas delgadas y largas, y 40 sedas cortas, espiniformes; escutelo con 6 sedas delgadas y largas, y 46 sedas cortas espiniformes; área respiratoria con 7 sedas delgadas y largas (Fig. 15). Segmento abdominal V, preescudo con 2 sedas delgadas y largas, y 38 sedas cortas, espiniformes; subescudo con 3 sedas largas y delgadas; escudo con 12 sedas delgadas y largas, y 40 sedas cortas, espiniformes; escutelo con 8 sedas delgadas y largas, y 42 sedas cortas espiniformes; área respiratoria con 7 sedas delgadas y largas. Segmento abdominal VI: preescudo con 2 sedas delgadas y largas, y 36 sedas cortas, espiniformes; subescudo con 3 sedas largas y delgadas; escudo con 12 sedas delgadas y largas, y 34 sedas cortas, espiniformes; escutelo con 8 sedas delgadas y largas, y 19 sedas cortas espiniformes; área respiratoria con 7 sedas delgadas y largas. Segmento abdominal VII: con 2 hileras transversales de sedas dorsales, una anterior con 4 sedas delgadas y largas, y 4 sedas cortas dispersas y la hilera posterior con 10 sedas delgadas y largas; área respiratoria con 7 sedas delgadas y largas. Segmento abdominal VIII: con 2 hileras transversales de sedas dorsales, una anterior con 2 sedas delgadas y largas; y la posterior con 8 sedas delgadas y largas, sedas cortas espiniformes ausentes; área respiratoria con 7 sedas delgadas y largas. Segmento abdominal IX: con 2 hileras transversales de sedas dorsales, una anterior con 8 sedas delgadas y largas, y 4 sedas cortas dispersas; y la hilera posterior con 8 sedas delgadas y largas. Segmento abdominal X: dorso con 68 sedas delgadas y largas mezcladas con 142 sedas cortas espiniformes; áreas laterales con 7 sedas delgadas y largas (Fig. 15). Raster sin palidia; campus con 6 sedas delgadas y cortas; teges formado por 80-81 sedas hamate; bárbula con 20-21 sedas delgadas y largas. Abertura anal transversa.

Pupa. Macho. Aspecto general (Fig. 16). Longitud 22.5 $\mathrm{mm}$, anchura máxima $11.0 \mathrm{~mm}$. Exarata. Cuerpo robusto, de forma oval alargada, amarillo rojizo, con una fina cubierta de microtrichia dorada más notable hacia el final del abdomen.

Cabeza. Fuertemente inclinada hacia abajo; piezas bucales claramente diferenciadas; frente con superficie irregular; clipeo convexo fusionado al labro; canthus ocular aparente; ojos hundidos, poco prominentes; tecae antenales engrosados, claramente visibles.

Tórax. Pronoto convexo, ligeramente elevado hacia los bordes laterales; meso y metanoto bien diferenciados; tecae elitrales más cortos que las tecae alares; las tecae elitrales se proyectan hasta la mitad del III segmento abdominal, las tecae alares se extienden hasta el V segmento abdominal. Abdomen. En vista dorsal se distinguen 10 segmentos, y en vista ventral sólo se observan 7 segmentos bien definidos. Con 5 pares de órganos dioneiformes bien definidos en el dorso de los segmentos I-II, II-III, III-IV, IV-V, V-VI. Lóbulos pleurales redondeados. Primer orificio respiratorio elongado, con un peritrema fino y cubierto por la teca alar; tubérculos respiratorios de los segmentos II-IV ovalados, prominentes, con un peritrema ligeramente esclerosado; orificios respiratorios de los segmentos V-VIII reducidos y cerrados. Dorso de los segmentos VII y IX con 2 fóveas dorso-laterales. Último segmento sin urogomphi ni lóbulos laterales, ápice ligeramente aguzado; ampula genital ligeramente pronunciada.

Material examinado. Veintidos larvas de tercer estadio y 8 pupas correlacionadas con los adultos, colectadas en COLOMBIA: Cundinamarca, Subachoque, Vereda Cánica Baja, Finca Potosí (455'56” N; 74¹0’36” O), $2610 \mathrm{~m}$, rizósfera de pasto Pennisetum clandestinum (Poaceae) 22-XI-2004, J. C. Neita, C. Zuluaga y D. 
Carrillo; mismos datos excepto: cultivo de papa Solanum tuberosum (Solanaceae); Bogotá (4³5'56” N; 7604'51", O) 2599 m, rizósfera de pasto $P$. clandestinum, 22-XI-2004, J. C. Neita; mismos datos excepto rizósfera de rosa Rosa sp. (Rosaceae); Mosquera, Finca Marengo-Universidad Nacional de Colombia (442’27” N; 74¹4'02” O) 2546 m, 23-XII-2002, C. Zuluaga (UNAB).

\section{Comentarios taxonómicos}

Las larvas de esta especie se diferencian de las demás larvas conocidas hasta el momento del género Ancognatha por la siguiente combinación de caracteres: haptomerum de la epifaringe bidentado; borde lateroposterior izquierdo de la epifaringe angulado; laeophoba con 18 sedas delgadas y cortas; en la mala los unci 2 y 3 están separados por una distancia 2 veces mayor al espacio que separa al primer unci del segundo; los 3 unci no están dispuestos en el mismo plano; las placas respiratorias del primer segmento abdominal son más pequeñas que en los segmentos II a VIII. Las pupas de $A$. ustulata y $A$. sellata son muy parecidas, sólo existen ligeras diferencias en las dimensiones corporales y apendiculares, así como en la densidad de la cubierta de microtrichia.

Biología. Los adultos tienen hábitos nocturnos y son atraídos por las fuentes de luz. Vuelan entre las 20:00 y 06:00 hrs. Los adultos emergen del suelo durante las primeras lluvias del año (marzo-junio). Las larvas pupan a una profundidad de $35-55 \mathrm{~cm}$. La pupa se desarrolla rodeada por la última exuvia larval. Las hembras, una vez se aparean, se entierran y ovopositan. Su ciclo de vida es anual. Las larvas se alimentan de materia orgánica en descomposición, y pueden ser abundantes en suelos con alto contenido de esta misma. Ante la escasez de materia orgánica, las larvas consumen material vivo, como tubérculos de papa, raíces de pasto, maíz y flores, por lo tanto podemos categorizarlas como rizófagas facultativas. Distribución. Esta especie se conoce de Colombia, Ecuador y Venezuela (Endrödi, 1966, 1985; Ratcliffe 2003). Endrödi (1985) la cita de México y Panamá, pero Morón et al.(1997) y Ratcliffe (2003) no confirmaron registros para esos países. Lo más probable es que la información de Endrödi esté basada en ejemplares incorrectamente rotulados. Ancognatha ustulata se distribuye en la cordillera de los Andes por encima de los 2600 metros. A elevaciones mayores (3200-3500 m) es posible encontrar en el mismo sitio a $A$. ustulata, $A$. scarabaeoides y $A$. lutea en los departamentos de Boyacá y Cundinamarca.

Material examinado. Especímenes adultos colectados: 56. COLOMBIA: Boyacá, Monguí (543’52”N; 72 51'
24”O) 2920 m, 4-IV-1997, T. Baquero; Moniquirá (5 52' 51'” ; 73 34' 45"O) 1050 m, 21-X-1997, Y. Aranda; Nobsa (5 46' 27’'N; 72 48' 45”O) 2610 m, 1-III-1999; S. C. Nuevo Colón (5 47' 04’’N; 7307' 16”O) 2517 m, 15IV-1997; Ávila Tocá (5³3'59”N; 73 11' 16”O) 2761 m, 17-X-2000; Corral, Paipa (5'47'04”N; 7307' 16”O) 2517 m, 5-II-1998; S. Subis. Paipa (5'21' 25’N; 7327' 36"O) 2465 m, 5-XI-1992, F. García. Cundinamarca, Albán (4 52' 46”N; 74²6' 25”O) 2251 m, 26-II-1976, R. Mariño; Bogotá (4 35' 56”N; 7404' 51'O) 2599 m, 28XI-1975, O. Moreno; mismos datos excepto 1-XII-1996, Hortua; igual excepto 11-I-1981, Vargas; Bojacá (4 44' 05”N; 74 20' 41”O) 2598 m, 22-IV-1994; S. C. Chía (4 51'49”N; 74 03' 48”O) 2562 m, 6-VI-1999, O. Higuera; R. Quevedo, Fomeque (4 29' 27'N; 7353' 54'O) 1895 m, 10-VIII-2001, R. Piamba; Y. Reyes, Cajicá (4 55' 11'N; 7401' 82"O) 2564 m, 24-IV-1993, Y. Barrera; La Calera (4 42' 27'N; 74 14' 02'” O) 2718 m, 22-IX-1992, J. Zuleta; Madrid (4 36' 58'N; 74 16' 02”' O) 2554 m, 11V-1990, E. Catuña; Mosquera (4 42' 27'N; 74 14'02'O) 2546 m, 27-III-1999; Y. P. Subachoque (4 $55^{\prime}$ 56”'N; 74º 10'36" O) 2610 m, 23-X-1997, F. Montes.

\section{Agradecimientos}

A la familia Carrillo Quiroz, al Ingeniero César Zuluaga e Ilán Garzón por su colaboración en el trabajo de campo. De igual manera a John Albeiro Quiroz (MEFLG), German Amat García (ICN-MHN-CO), Pilar Hernández (CE-CIAT), Freddy Calvache (CE-UQ), Carmen Eliza Posso y Nancy Carrejo (MEUV), Gonzalo Andrade (MHN-GA), Alberto Varela (CE-FA), Sandra Restrepo (CE-UC), Martha Wolff (CE-UDA), Rodrigo Torres (CEUPN), Celsa García (UNAB), Alex Mauricio Jiménez (MHN-CE), Astrid Pulido y Mónica Ospina (IAVH). A Hortensia Carrillo Ruiz por su apoyo durante la estancia del primer autor en Xalapa, Veracruz, México. En parte, este trabajo muestra resultados del proyecto "Plagas subterráneas de América del Sur" (Centro Internacional de Agricultura Tropical (CIAT) y la Agencia Alemana (AGZ) que estuvo a cargo del Dr. Andreas Gaigl. También es una contribución al proyecto "Coleópteros lamelicornios de América Latina" apoyado por el Instituto de Ecología, A.C., Xalapa (cuenta 2000910011).

\section{Literatura citada}

Böving, A. 1942. A classification of larvae and adults of the genus Phyllophaga (Coleoptera: Scarabaeidae). Memories of the Entomological Society of Washington, No. 2:1-95.

Endrödi, S. 1966. Monographie der Dynastinae (Coleoptera, 
Lamellicornia). I. Teil. Entomologische Abhandlungen 33:1460.

Endrödi, S. 1985. The Dynastinae of the world. Dr. W. Junk Publ., Dordrecht. 800 pp.

Krajcik, M. 2005. Dynastinae of the world. Checklist. (Coleoptera: Scarabaeidae: Dynastinae). Animma 10, supplement 2:1122.

Londoño, M. E. y M. Pérez. 1994. Reconocimiento de los enemigos naturales de la chisa o mojojoy (Coleoptera: Scarabaeidae) en el oriente antioqueño. Revista Colombiana de Entomología. 20:199-206.

Montoya, G. y A. Madrigal. 1994. Evaluación de trampas de luz para el control de adultos de Scarabaeidae en cultivos de papa en La Unión (Antioquia). Revista Colombiana de Entomología. 20:130-136.

Morón, M. A. 1986. El género Phyllophaga en México. Morfología, distribución y sistemática supraespecífica (Insecta: Coleoptera). Publicación 20. Instituto de Ecología, Xalapa, Veracruz. 341 p.

Morón, M. A. 1987. Los estados inmaduros de Dynastes hyllus Chevrolat (Coleoptera: Melolonthidae: Dynastinae) con observaciones sobre su biología y el crecimiento alométrico del imago. Folia Entomológica Mexicana 72:33-74.

Morón, M.A. 1993. Observaciones comparativas sobre la morfología pupal de los Coleoptera Melolonthidae neotropicales. Giornale italiano di Entomologia 6:249-255.

Morón, M. A., B. C. Ratcliffe y C. Deloya. 1997. Atlas de los escarabajos de México, Coleoptera Lamellicornia, vol. I, familia Melolonthidae. Sociedad Mexicana de Entomología, y Comisión Nacional para el Conocimiento y Uso de la Biodiversidad (CONABIO), México, D.F.. 280 p.

Otoya, F. J. 1945. Anotaciones sobre el género Ancognatha y descripción de una nueva especie (Scarabaeidae). Caldasia 3:273-282

Pardo-Locarno, L. C., P. Franco y A. Alarcón. 1993. Contribución al conocimiento de las «chisas» (Coleoptera-Scarabaeiodea) de San Antonio-Cauca, Colombia. In Diversidad y manejo de plagas subterráneas, M. A. Morón (comp.). Publicación especial de la Sociedad Mexicana de Entomología e Instituto de Ecología, Xalapa, Veracruz. p. 91-104.

Pardo-Locarno, L. C., M. A. Morón, A. Gaigl y A. C. Belloti. 2003. Los complejos regionales de Melolonthidae (Coleoptera) rizófagos en Colombia. In Estudios sobre coleópteros del suelo en América, A. Aragón, M. A. Morón y A. Marín (eds.).
Publicación especial Benemérita Universidad Autónoma de Puebla, Puebla. p. 45-66.

Pardo-Locarno, L. C., R. González y J. Montoya. 2006. Description of a new species and new country records of Ancognatha Erichson (Coleoptera: Scarabaeidae: Dynastinae) from Colombia. Zootaxa 1139:63-68.

Ramírez-Salinas, M. A. Morón y A. E. Castro-Ramírez. 2004. Descripción de los estados inmaduros de tres especies de Anomala, Ancognatha y Ligyrus (Coleoptera: Melolonthidae: Rutelinae y Dynastinae) con observaciones de su biología. Acta Zoológica Mexicana (nueva serie) 20:67-82

Ratcliffe, B. 1992. A new species of Ancognatha from Panama (Coleoptera : Scarabaeidae: Dynastinae). The Coleopterists Bulletin 46:256-259.

Ratcliffe, B. C. 2003. The dynastine scarab beetles of Costa Rica and Panama (Coleoptera: Scarabaeidae: Dynastinae). Bulletin of the University of Nebraska State Museum 16:1-506.

Restrepo-Giraldo, H. y A. López-Ávila, 2000. Especies de chisas (Coleoptera: Melolonthidae) de importancia agrícola en Colombia. Corporación Colombiana de Investigación Agropecuaria-Corpoica and Programa Nacional de Manejo Integrado de Plagas-MIP, Bogotá. 62 p.

Restrepo-Giraldo, H., M. A. Morón, F. Vallejo, L. C. PardoLocarno y A. López-Ávila. 2003. Catálogo de Coleoptera Melolonthidae (Scarabaeidae Pleurosticti) de Colombia. Folia Entomológica Mexicana 42: 239-263

Ritcher, R. O. 1966. White grubs and their allies. Oregon State University Press, Corvallis. p. 219.

Ruiz, B. N. y F. Posada. 1985. Aspectos biológicos de las chisas en la sabana de Bogotá. Revista Colombiana de Entomología 11:21-26.

Sánchez-Gutiérrez, G. y N. C. Vásquez-Acosta, 1996. Manejo de plagas en arracacha. Corporación Colombiana de Investigación Agropecuaria-Corpoica, Instituto Colombiano Agropecuario-ICA, Fondo de Desarrollo Rural IntegradoDRI, y Alcaldía Municipal de Cajamarca, Ibaqué. 43 p.

Stehr, F. W. (ed.). 1987. Immature insects, vol. 1. Kendall-Hunt Publications, Dubuque, Iowa. $450 \mathrm{p}$.

Vallejo, F. 1997. Contribución al conocimiento de las plagas subterráneas-chisas (Coleoptera: Melolonthidae) del Oriente de Antioquia-Colombia. Tesis maestría, Facultad de Ciencias, Universidad Nacional de Colombia, Sede de Medellín. 235 p. 